\title{
THIN INTERPOLATING SEQUENCES AND THREE ALGEBRAS OF BOUNDED FUNCTIONS
}

\author{
HÅKAN HEDENMALM
}

\begin{abstract}
We consider the closed subalgebra $\mathbf{A}$ of $H^{\infty}$ generated by the thin interpolating Blaschke products, the smallest $C^{*}$ subalgebra $\mathbf{B}$ of $L^{\infty}$ containing $\mathbf{A}$, and the Douglas algebra $\mathbf{E}$ generated by the complex conjugates of thin interpolating Blaschke products. Our main result is that every $\mathbf{E}$-invertible inner function is a finite product of thin interpolating Blaschke products, making $\mathbf{B}=C_{\mathbf{E}}$. We apply results of Chang and Marshall to prove that $\mathbf{A}=\mathbf{B} \cap H^{\infty}$, that finite convex combinations of finite products of thin interpolating Blaschke products are dense in the closed unit ball of $\mathbf{A}$, and that the corona theorem holds for $\mathbf{A}$.
\end{abstract}

0. Introduction. $\mathbf{D}$ will always be the open unit disc $\{z \in \mathbf{C}:|z|<1\}$, and $\mathbf{T}=\partial \mathbf{D}$ will be the unit circle $\{z \in \mathbf{C}:|z|=1\} . H^{\infty}=H^{\infty}(\mathbf{D})$ is the Banach algebra of bounded analytic functions on $\mathbf{D}$, supplied with the uniform norm. It is well known that we can regard $H^{\infty}$ as a closed subalgebra of $L^{\infty}=L^{\infty}(\mathbf{T})$ via nontangential boundary values.

A sequence $\left\{z_{n}\right\} \subset \mathbf{D}$, finite or infinite, is an $\left(H^{\infty}\right)$ interpolating sequence if every interpolation problem

$$
f\left(z_{n}\right)=a_{n} \text { for all } n
$$

with $\left\{a_{n}\right\}$ bounded, has solution $f \in H^{\infty}$. Clearly, every finite sequence that does not contain the same point twice is an interpolating sequence. A famous theorem of Lennart Carleson (see Carleson [1958], Garnett [1981, Chapter VII]) states that a sequence $\left\{z_{n}\right\}_{0}^{\infty} \subset \mathbf{D}$ is interpolating if and only if

$$
\prod_{k, k \neq n} \rho\left(z_{k}, z_{n}\right) \geq \delta, \quad n=0,1,2, \ldots
$$

for some constant $\delta>0$. Here $\rho$ denotes the pseudohyperbolic metric

$$
\rho(z, \varsigma)=|(z-\varsigma) /(1-\bar{\zeta} z)|, \quad z, \varsigma \in \mathbf{D} .
$$

Call an interpolating sequence $\left\{z_{n}\right\}$ thin if it is finite or

$$
\prod_{k, k \neq n} \rho\left(z_{k}, z_{n}\right) \rightarrow 1 \quad \text { as } n \rightarrow \infty .
$$

By a recent joint paper by Carl Sundberg and Thomas Wolff [1983], a sequence is thin interpolating if and only if it is an interpolating sequence for the uniform algebra $Q A=H^{\infty} \cap \operatorname{VMO}(\mathbf{T})$, where $\operatorname{VMO}(\mathbf{T})$ is the space of functions on $\mathbf{T}$ with vanishing mean oscillation.

Received by the editors August 6, 1985 and, in revised form, January 20, 1986.

1980 Mathematics Subject Classification (1985 Revision). Primary 46J15; Secondary 46J10, $30 \mathrm{H} 05$. 
A Blaschke product associated to a thin interpolating sequence is called a thin interpolating Blaschke product. It should be pointed out that in our terminology, a Blaschke product multiplied by a unimodular constant is also a Blaschke product.

Let $\mathbf{A}$ be the closed subalgebra of $H^{\infty}$ generated by the thin interpolating Blaschke products, and let $\mathbf{B}=[\mathbf{A}, \overline{\mathbf{A}}]$ be the smallest (closed) $C^{*}$ subalgebra of $L^{\infty}$ containing $\mathbf{A}$. In other words, $\mathbf{B}$ is generated by ratios of thin interpolating Blaschke products. $\mathbf{E}=\left[H^{\infty}, \overline{\mathbf{A}}\right]$ will be the Douglas algebra (for a definition, see Garnett [1981]) generated by $H^{\infty}$ and the complex conjugates of all thin interpolating Blaschke products.

Our main result is that every $\mathbf{E}$-invertible inner function is in fact a finite product of thin interpolating Blaschke products, and hence $\mathbf{B}=C_{\mathbf{E}}$. Here $C_{\mathbf{E}}$ denotes the $C^{*}$ algebra generated by the $\mathbf{E}$-invertible inner functions and their complex conjugates. Another consequence is that the finite products of thin interpolating Blaschke products are the only inner functions there are in $\mathbf{B} \cap H^{\infty}$, and hence by Theorem 4.1 in the joint paper [1977] by Sun-Yung Chang and Donald Marshall, $\mathbf{A}=\mathbf{B} \cap H^{\infty}$, and finite convex combinations of finite products of thin interpolating Blaschke products are dense in the closed unit ball of $\mathbf{A}$. There are some other properties of $\mathbf{A}, \mathbf{B}$, and $\mathbf{E}$ which follow from the results stated in Chang-Marshall [1977]; for example, $\mathbf{A}$ has the corona property, that is, $\mathbf{D}$ is dense in the maximal ideal space $\mathcal{M}(\mathbf{A})$ of $\mathbf{A}$.

The author would like to mention the following related open problem, which has been posed by Peter Jones [1981, p. 320] and John Garnett [1981, p. 430; 1984]: Do the interpolating Blaschke products generate $H^{\infty}$ ?

1. Basic concepts. Let $\mathcal{M}\left(H^{\infty}\right)$ be the maximal ideal space of $H^{\infty}$, that is, the space of all (nonzero) complex homomorphisms on $H^{\infty}$, provided with the Gelfand topology. The famous corona theorem states that $\mathbf{D}$ is dense in $\mathcal{M}\left(H^{\infty}\right)$. For $f \in H^{\infty}$, its Gelfand transform $\hat{f}$ is a continuous function on $\mathcal{M}\left(H^{\infty}\right)$ which extends $f$. When it cannot cause any confusion, we will usually omit the distinction between a function and its Gelfand transform.

The Gleason parts are the connectivity components of $\mathcal{M}\left(H^{\infty}\right)$ when endowed with the norm topology of the dual Banach space $\left(H^{\infty}\right)^{*}$ (Hoffman $[\mathbf{1 9 6 7}$, p. 103]). For instance, $\mathbf{D}$ is a Gleason part of $\mathcal{M}\left(H^{\infty}\right)$. Let $\mathcal{P}(m)$ denote the Gleason part containing the point $m \in \mathcal{M}\left(H^{\infty}\right)$.

For $\varsigma \in \mathbf{D}$, let

$$
L_{\zeta}(z)=(z+\varsigma) /(1+\bar{\zeta} z), \quad z \in \mathbf{D},
$$

which maps D homeomorphically onto itself. According to Hoffman [1967] (see also Garnett [1981, Chapter X]), we can introduce for every $m \in \mathcal{M}\left(H^{\infty}\right)$ an analytic mapping $L_{m}: \mathbf{D} \rightarrow \mathcal{M}\left(H^{\infty}\right)$ which varies continuously with $m$ such that $L_{m}(0)=m, L_{m}(\mathbf{D})=\mathcal{P}(m)$, and if $m=\varsigma \in \mathbf{D}, L_{m}=L_{\zeta} . L_{m}$ is constant if and only if $m$ is not in the closure of any interpolating sequence. We define $\mathcal{F}$ to be the set of all points in $\mathcal{M}\left(H^{\infty}\right)$ which are in the closure of a thin interpolating sequence. Proposition 2.2 tells us that $\mathcal{F}$ is the union of a family of nontrivial Gleason parts, and by Proposition 2.3, $L_{m}$ is a homeomorphism for $m \in \mathcal{F}$, a fact which is well known (see Hoffman [1967, pp. 106-108]).

We should mention that is is customary to identify the Silov boundary of $\mathcal{M}\left(H^{\infty}\right)$ with $\mathcal{M}\left(L^{\infty}\right)$, the maximal ideal space of $L^{\infty}$. 
2. Results. We shall need the following lemma.

LEMMA 2.1. Let $\left\{z_{n}\right\}_{0}^{\infty}$ be a thin interpolating sequence. If the sequence $\left\{\zeta_{n}\right\}_{0}^{\infty}$ does not contain the same point twice and $\rho\left(z_{n}, \varsigma_{n}\right) \leq r$ for all $n$ for some $r<1$, the sequence $\left\{\zeta_{n}\right\}_{0}^{\infty}$ is also thin interpolating.

ProOF. Since $\left\{z_{n}\right\}_{0}^{\infty}$ is a thin interpolating sequence, there is a positive number $\delta(N)$ tending to 1 as $N \rightarrow \infty$, such that

$$
\prod_{n, n \geq N, n \neq k} \rho\left(z_{n}, \zeta_{n}\right) \geq \delta(N) \quad \text { for all } k \geq N .
$$

By a lemma of Garnett [1981, Lemma VII.5.3],

$$
\prod_{n, n \geq N, n \neq k} \rho\left(\zeta_{n}, \zeta_{k}\right) \geq\left(\delta(N)-2 r /\left(1+r^{2}\right)\right)\left(1-2 r \delta(N) /\left(1+r^{2}\right)\right)^{-1}
$$

for all $k \geq N$ if $N$ is sufficiently large. Since $\lim _{k \rightarrow \infty} \rho\left(\zeta_{n}, \zeta_{k}\right)=1$ for every $n$ (use Lemma I.1.4 of Garnett [1981]),

$$
\begin{aligned}
\liminf _{k \rightarrow \infty} \prod_{n, n \neq k} \rho\left(\varsigma_{n}, \zeta_{k}\right) & =\liminf _{k \rightarrow \infty} \prod_{n, n \geq N, n \neq k} \rho\left(\varsigma_{n}, \varsigma_{k}\right) \\
& \geq\left(\delta(N)-2 r /\left(1+r^{2}\right)\right)\left(1-2 r \delta(N) /\left(1+r^{2}\right)\right)^{-1}
\end{aligned}
$$

again if $N$ is sufficiently large. Letting $N \rightarrow \infty$ and thus $\delta(N) \rightarrow 1$, the right-hand side expression tends to 1 , and the assertion follows.

As a consequence, we have the following

PROPOSITION 2.2. $\mp$ is the union of a family of nontrivial Gleason parts.

ProOF. By the definition of $\mathcal{F}$, every point in $\mathcal{F}$ belongs to a nontrivial Gleason part. So, let $m_{0} \in \mathcal{F}$ be arbitrary, and suppose $m \in \mathcal{P}\left(m_{0}\right)$. It suffices to show that $m \in \mathcal{F}$. By assumption, there exists a thin interpolating sequence $\left\{z_{n}\right\}_{0}^{\infty}$ such that $m_{0} \in \overline{\left\{z_{n}\right\}_{o}^{\infty}}$, and thus there is a net $\left\{z_{n(\alpha)}\right\}_{\alpha}$ converging to $m_{0}$. Since $m$ belongs to $P\left(m_{0}\right)$, there exists a $\varsigma \in \mathbf{D}$ such that $\lim _{\alpha} L_{z_{n(\alpha)}}(\varsigma)=L_{m_{0}}(\varsigma)=m$. But by Lemma 2.1 , the sequence

$$
\varsigma_{n}=L_{z_{n}}(\varsigma)=\left(\varsigma+z_{n}\right) /\left(1+\bar{z}_{n} \varsigma\right), \quad n=0,1,2, \ldots,
$$

is also thin interpolating since $\rho\left(\zeta_{n}, z_{n}\right)=|\zeta|<1$, and because $\lim _{\alpha} \zeta_{n(\alpha)}=m$, the assertion follows.

Proposition 2.3. A thin interpolating Blaschke product $b$ has modulus 1 on those Gleason parts of $\mathcal{M}\left(H^{\infty}\right)$ which do not contain a point in the zero set $Z(\hat{b})=$ $\left\{m \in \mathcal{M}\left(H^{\infty}\right): \hat{b}(m)=0\right\}$. Since $Z(\hat{b})$ equals the closure of $Z(\hat{b}) \cap \mathbf{D}$ and $₹$ is the union of a family of Gleason parts, $\hat{b}$ has in particular modulus 1 on $\mathcal{M}\left(H^{\infty}\right) \backslash \mathcal{F}$. Also, no two points in $Z(\hat{b}) \backslash \mathbf{D}$ are contained in the same Gleason part of $\mathcal{F}$. Indeed, for every $m \in Z(\hat{b}) \backslash \mathbf{D}$ there is $a \theta \in \mathbf{R}$ such that

$$
\hat{b} \circ L_{m}(z)=e^{i \theta} z, \quad z \in \mathbf{D} \text {. }
$$

ProOF. The assertion is trivial if $b$ is a finite Blaschke product. So, let $\left\{z_{n}\right\}_{0}^{\infty}$ be the zeros of $b$. That $Z(\hat{b})$ equals the closure of $Z(\hat{b}) \cap \mathbf{D}=\left\{z_{n}\right\}_{0}^{\infty}$ follows from Lemma IX.3.3 in Garnett [1981]. Since $b$ is thin interpolating,

$$
\left|\left(b \circ L_{z_{n}}\right)^{\prime}(0)\right|=\left(1-\left|z_{n}\right|^{2}\right)\left|b^{\prime}\left(z_{n}\right)\right| \rightarrow 1
$$


as $n \rightarrow \infty$, and hence $\left|\left(b \circ L_{m}\right)^{\prime}(0)\right|=1$ for all $m \in Z(\hat{b}) \backslash \mathbf{D}$. On the other hand, $\left\|\hat{b} \circ L_{m}\right\| \leq 1$, and Schwarz' lemma now tells us that

$$
\hat{b} \circ L_{m}(z)=e^{i \theta} z, \quad z \in \mathbf{D},
$$

for all $m \in Z(\hat{b}) \backslash \mathbf{D}$ with a $\theta \in \mathbf{R}$ depending on $m$.

It remains to show that $\hat{b}$ has modulus 1 on those Gleason parts of $\mathcal{M}\left(H^{\infty}\right)$ which do not contain a point in $Z(\hat{b})$. To this end, let $m \in \mathcal{M}\left(H^{\infty}\right) \backslash \bigcup_{m_{0} \in Z(\hat{b})} \mathcal{P}\left(m_{0}\right)$ be arbitrary; by the corona theorem, there is a net $\left\{\zeta_{\alpha}\right\}_{\alpha}$ in $\mathbf{D}$ which converges to $m$. Our first step is to show that

$$
\liminf _{\alpha} \rho\left(\zeta_{\alpha}, z_{n}\right)=1 \text {. }
$$

If this were not true, there would be a (cofinal) subnet $\{\beta\}$ of $\{\alpha\}$, an $n(\beta) \in \mathbf{N}$ for every $\beta$, and an $\varepsilon, 0<\varepsilon<1$, such that (see Garnett [1981, p. 401])

$$
\rho\left(\varsigma_{\beta}, z_{n(\beta)}\right)=\sup \left\{\left|f\left(\varsigma_{\beta}\right)\right|: f \in H^{\infty},\|f\| \leq 1, f\left(z_{n(\beta)}\right)=0\right\} \leq \varepsilon
$$

for all $\beta$, and, consequently,

$$
\sup \left\{\left|f\left(\varsigma_{\beta}\right)-f\left(z_{n(\beta)}\right)\right|: f \in H^{\infty},\|f\| \leq 1, f\left(z_{n(\beta)}\right)=0\right\} \leq 2 \varepsilon<2
$$

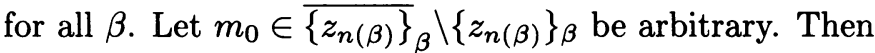

$$
\left|\hat{f}(m)-\hat{f}\left(m_{0}\right)\right| \leq \sup _{\beta}\left|f\left(\varsigma_{\beta}\right)-f\left(z_{n(\beta)}\right)\right| \leq 2 \varepsilon<2
$$

for all $f \in H^{\infty}$ with $\|f\| \leq 1$. Hence $m$ and $m_{0}$ lie in the same Gleason part, which is clearly impossible. Thus (2.1) follows.

Now the time has come to show that

$$
|\hat{b}(m)|=\lim _{\alpha}\left|b\left(\zeta_{\alpha}\right)\right|=1
$$

Observe that in order to show that (2.2) holds, we may assume without loss of generality that

$$
\inf _{n}\left(1-\left|z_{n}\right|^{2}\right)\left|b^{\prime}\left(z_{n}\right)\right|=\inf _{n} \prod_{k, k \neq n} \rho\left(z_{k}, z_{n}\right)=\delta,
$$

where $\delta \in(0,1)$ is arbitrarily close to 1 , by dividing $b$ by finitely many of its Blaschke factors, since all finite Blaschke products satisfy (2.2). Choose $\delta$ so that $r(\delta)$ of Lemma X.1.4 in Garnett $[\mathbf{1 9 8 1}]$ is close to 1 , and let $\lambda(\delta)$ be as prescribed in that lemma. Since

$$
\lim _{\alpha} \inf _{n} \rho\left(\zeta_{\alpha}, z_{n}\right)=1,
$$

there is an index $\alpha_{0}(\delta)$ such that

$$
\inf _{n} \rho\left(\varsigma_{\alpha}, z_{n}\right) \geq \lambda(\delta) \text { for all } \alpha \geq \alpha_{0}(\delta),
$$

that is,

$$
\zeta_{\alpha} \in \mathbf{D} \backslash \bigcup_{n} U_{n} \quad \text { for } \alpha \geq \alpha_{0}(\delta),
$$

where $U_{n}=\left\{z \in \mathbf{D}: \rho\left(z, z_{n}\right)<\lambda(\delta)\right\}$. Hence by Lemma X.1.4 in Garnett [1981],

$$
\left|b\left(\varsigma_{\alpha}\right)\right| \geq r(\delta) \text { for all } \alpha \geq \alpha_{0}(\delta) .
$$


Since $r(\delta)$ was close to 1 , we conclude that $\lim _{\alpha}\left|b\left(\varsigma_{\alpha}\right)\right|=1$. The proof of the proposition is complete.

It is a consequence of Proposition 2.3 that $\mathcal{F}$ is an open subset of $\mathcal{M}\left(H^{\infty}\right)$. If $\mathcal{M}(\mathbf{E})$ denotes the maximal ideal space of the Douglas algebra $\mathbf{E}$, Proposition 2.3 has the following corollary when combined with Theorem IX.1.3 in Garnett [1981].

Corollary 2.4. $\mathcal{M}(\mathbf{E})=\mathcal{M}\left(H^{\infty}\right) \backslash \mathcal{F}$.

By the Douglas-Rudin theorem,

$$
\left[H^{\infty}, \overline{H^{\infty}}\right]=L^{\infty}
$$

and since the set $\mathcal{M}\left(H^{\infty}\right) \backslash\left(\mathcal{F} \cup \mathcal{M}\left(L^{\infty}\right)\right)$ is nonempty (there are, for example, onepoint Gleason parts not contained in the Silov boundary $\left.\mathcal{M}\left(L^{\infty}\right)\right)$, Corollary 2.4 has the following consequence.

COROllary 2.5. A is a proper subalgebra of $H^{\infty}$.

We are now ready to present our main result.

THEOREM 2.6. Every E-invertible inner function is a finite product of thin interpolating Blaschke products.

ProOF. Let $u$ be an arbitrary E-invertible inner function, and denote by $Z(\hat{u})$ the zero set $\left\{m \in \mathcal{M}\left(H^{\infty}\right): \hat{u}(m)=0\right\}$. Our first step will be to show that the zeros of $u$, when counted with respect to multiplicity, form a finite union of thin interpolating sequences.

By Theorem IX.1.3 in Garnett $[\mathbf{1 9 8 1}],|\hat{u}(m)|=1$ on $\mathcal{M}(\mathbf{E})=\mathcal{M}\left(H^{\infty}\right) \backslash \mathcal{F}$, and consequently, $Z(\hat{u}) \subset \mathcal{F}$.

Introduce $\varepsilon, 0<\varepsilon<1$, to be determined later. For every thin interpolating Blaschke product $b$, let

$$
\Omega_{\varepsilon}(b)=\left\{m \in \mathcal{M}\left(H^{\infty}\right):|\hat{b}(m)|<\varepsilon\right\} .
$$

Since $Z(\hat{u}) \subset \mathcal{F}$, a compactness argument provides us with finitely many thin interpolating Blaschke products $b_{1}, \ldots, b_{n(\varepsilon)}$ such that

$$
Z(\hat{u}) \subset \bigcup_{k=0}^{n(\varepsilon)} \Omega_{\varepsilon}\left(b_{k}\right)
$$

If $\left\{z_{j}\left(b_{k}\right)\right\}_{j=0}^{\infty}$ are the zeros of $b_{k}$, let $\delta, 0<\delta<1$, equal

$$
\delta=\min _{1 \leq k \leq n(\varepsilon)} \inf _{j}\left(1-\left|z_{j}\left(b_{k}\right)\right|^{2}\right)\left|b_{k}^{\prime}\left(z_{j}\left(b_{k}\right)\right)\right|,
$$

and put $\varepsilon=r(\delta)$, where $r(\delta)$ is as in Lemma X.1.4 in Garnett [1981]. According to that lemma, there exists a $\lambda(\delta), 0<\lambda(\delta)<1$, such that

$$
\Omega_{\varepsilon}\left(b_{k}\right) \cap \mathbf{D} \subset \bigcup_{j=0}^{\infty} \Delta_{j, k}, \quad k=1, \ldots, n(\varepsilon),
$$

where $\Delta_{j, k}$ is the $\operatorname{disc}\left\{z \in \mathbf{D}: \rho\left(z, z_{j}\left(b_{k}\right)\right)<\lambda(\delta)\right\}$.

We now claim that the number of zeros of $u$ in $\Delta_{j, k}$ (counted with respect to multiplicity, as always) has a bound that is independent of $j$ and $k$. Once this is 
established, Lemma 2.1 will tell us that the zeros of $u$, when counted with respect to multiplicity, form a finite union of thin interpolating sequences.

We argue by contradiction. So, assume that there is a $k, 1 \leq k \leq n(\varepsilon)$, and an increasing sequence $\left\{j_{n}\right\}_{n=0}^{\infty}$ of positive integers such that the number of zeros of $u$ in $\Delta_{j_{n}, k}$ tends to infinity as $n \rightarrow \infty$. Then $\hat{u}(m)=0$ on $\mathcal{P}\left(m_{0}\right)$ for an arbitrary cluster point $m_{0} \in \mathcal{M}\left(H^{\infty}\right) \backslash \mathbf{D}$ of the sequence $\left\{z_{j_{n}}\left(b_{k}\right)\right\}_{j=0}^{\infty}$. But then $\bigcup_{k=0}^{n(\varepsilon)} \Omega_{\varepsilon}\left(b_{k}\right)$ would cover the closure of $P\left(m_{0}\right)$, which is impossible in view of Proposition 2.3.

From what we have done so far, it follows that $u=b v$, where $b$ is a finite product of thin interpolating Blaschke products, and $v$ is a singular inner function. Our final step is to show that $v$ equals a unimodular constant.

Since $u$ is $\mathbf{E}$-invertible, $v$ is, too. Unless $v$ is a unimodular constant, $v$ is not invertible in $H^{\infty}$. So, assuming that $v$ is not invertible in $H^{\infty}$, we find an $m \in \mathcal{F}$ such that $\hat{v}(m)=0$. But since every positive power $v^{\alpha}, \alpha>0$, of $v$ belongs to $H^{\infty}$, $\hat{v}$ must vanish identically on the closure of the analytic disc $P(m)$. But we already encountered such a situation in this proof-with $u$ instead of $v$, though-and in exactly the same fashion, we obtain a contradiction. The proof of the theorem is complete.

If $C_{\mathbf{E}}$ denotes the $C^{*}$ algebra generated by the $\mathbf{E}$-invertible inner functions and their complex conjugates, Theorem 2.6 has the following corollary.

COROllary 2.7. $C_{\mathrm{E}}=\mathbf{B}$.

COROLLARY 2.8. Let $b$ be a finite product of thin interpolating Blaschke products. If $f \in H^{\infty},\|f\|<1$, is such that $\bar{f} b$ equals an $H^{\infty}$ function $g$ almost everywhere on $\mathbf{T}$, then the function

$$
b_{f}(z)=(b(z)-f(z)) /(1-g(z)), \quad z \in \mathbf{D},
$$

is a finite product of thin interpolating Blaschke products.

PROOF. The assertion is immediate from Theorem 2.6, since $b_{f}$ is an $\mathbf{E}$-invertible inner function.

REMARK 2.9. One can show that if $b$ is an $n$-fold product of thin interpolating Blaschke products times a finite Blaschke product, then $b_{f}$ is also an $n$-fold product of thin interpolating Blaschke products times a finite Blaschke product.

Chang and Marshall showed in $[\mathbf{1 9 7 7}]$ that for an arbitrary Douglas algebra $B$, the closed unit ball of $H^{\infty} \cap C_{B}$ is the norm-closed convex hull of the Blaschke products in $H^{\infty} \cap C_{B}$. Here $C_{B}$ denotes the $C^{*}$ algebra generated by the $B$ invertible inner functions and their complex conjugates. They also showed that $B=H^{\infty}+C_{B}$, and that $\mathbf{D}$ is dense in the maximal ideal space of $H^{\infty} \cap C_{B}$. In our case $B=\mathbf{E}$, their results specialize to prove the following. Observe that an inner function in $\mathbf{B} \cap H^{\infty}$ is $\mathbf{E}$-invertible.

COROllary 2.10. (a) $\mathbf{A}=\mathbf{B} \cap H^{\infty}$, and finite convex combinations of finite products of thin interpolating Blaschke products are dense in the closed unit ball of A,

(b) $\mathbf{E}=H^{\infty}+\mathbf{B}$,

(c) $\mathbf{D}$ is dense in the maximal ideal space $\mathcal{M}(\mathbf{A})$ of $\mathbf{A}$.

3. Acknowledgments. The author has the pleasure to thank the Department of Computer Science at Uppsala University for its assistance with the computer processing of this manuscript, and the referee for his helpful comments. 
ADDED IN PROOF. After this paper was accepted for publication, I received an unpublished note from Keiji Izuchi entitled Interpolating and sparse Blaschke products, in which he also obtains Theorem 2.6.

\section{REFERENCES}

L. Carleson, [1958] An interpolation problem for bounded analytic functions, Amer. J. Math. 80, 921930.

S.-Y. Chang and D. E. Marshall, [1977] Some algebras of bounded analytic functions containing the disk algebra, Banach Spaces of Analytic Functions, Lecture Notes in Math., vol. 604, SpringerVerlag, Berlin, Heidelberg, and New York, pp. 12-20.

J. B. Garnett, [1981] Bounded analytic functions, Academic Press, New York, 1981.

, [1984] Some open problems concerning $H^{\infty}$ and BMO, Linear and Complex Analysis Problem Book, Lecture Notes in Math., vol. 1043, Springer-Verlag, Berlin, Heidelberg, New York, and Tokyo, pp. 330-332.

K. Hoffman, [1967] Bounded analytic functions and Gleason parts, Ann. of Math. (2) 86, 74-111.

P. W. Jones, [1981] Ratios of interpolating Blaschke products, Pacific J. Math. 95, 311-321.

C. Sundberg and T. H. Wolff, [1983] Interpolating sequences for $Q A_{B}$, Trans. Amer. Math. Soc. 276, 551-581.

Department of MAThematics, Uppsala University, S-752 38 UpPSAla, SWEdEN 48109

Current address: Department of Mathematics, University of Michigan, Ann Arbor, Michigan 